\title{
Evaluating the effect of Matricaria chamomilla and Melissa officinalis on pain intensity and satisfaction with pain management in patients after orthopedic surgery
}

\author{
Reza Saidi $^{1^{\complement}}$, Haydeh Heidari ${ }^{2^{*}}{ }^{\circledR}$ Morteza Sedehi $^{3^{\circledR}}$, Behrouz Safdarian $^{4}$ \\ ${ }^{1}$ Medical Surgical Nursing, Faculty of Nursing and Midwifery, Shahrekord University of Medical Sciences, Shahrekord, Iran \\ ${ }^{2}$ Faculty of Nursing and Midwifery, Modeling in Health Research Center, Shahrekord University of Medical Sciences, Shahrekord, Iran \\ ${ }^{3}$ Modeling in Health Research Center and Faculty of Public Health, Department of Epidemiology and Biostatistics, Shahrekord University of Medical \\ Sciences, Shahrekord, Iran \\ ${ }^{4}$ Orthopedic Specialist, Medical School, Shahrekord University of Medical Sciences, Shahrekord, Iran
}

\section{A R T I C L E I N F O}

\section{Article Type:}

Original Article

\section{Article History:}

Received: 7 January 2020

Accepted: 6 April 2020

\section{Keywords:}

Pain

Satisfaction with pain management Melissa officinalis

Matricaria chamomilla

\begin{abstract}
A B S T R A C T
Introduction: The most important compliant of post-operative orthopedic patients is severe and intolerable pain. This pain has negative impacts on patient's satisfaction with pain management and bone healing after surgery. This study was conducted with the aim of evaluating the effects of Melissa officinalis (Melissa) tea and Matricaria chamomilla (Chamomile) tea on pain intensity and satisfaction with pain management in patients after lower limb orthopedic surgery.

Methods: This was a randomized controlled trial conducted on 96 patients after lower limb orthopedic surgery in Ayatollah Kashani hospital of Shahrekord city, Iran. Patients were randomized to M. officinalis, Chamomile and control groups with blocking randomization method. For M. officinalis group, $1 \mathrm{~g}$ of Melissa tea, for Chamomile group $1 \mathrm{~g}$ chamomile tea and for control group black tea was prescribed 3 times a day. Study variables were evaluated before and $30 \mathrm{~min}$ after each stage of intervention and then the mean changes of pain intensity and satisfaction before interventions and after each step of intervention were compared between groups. Significance level was considered as $P<0.05$.

Results: Before intervention, the mean scores of pain intensity and satisfaction with pain management had no significance difference between groups. The mean changes of pain intensity and satisfaction with pain management (after first 3 steps of intervention) in M. officinalis and $M$. chamomilla groups were significantly greater than the ones in control group $(P<0.05)$. Conclusion: Prescription of M. officinalis tea or Chamomile tea might be used as effective remedy for reducing pain and increasing satisfaction with pain management after orthopedic surgery.
\end{abstract}

Implication for health policy/practice/research/medical education:

This research showed the positive effects of Melissa officinalis and Matricaria chamomilla on pain intensity and satisfaction with pain management in patients after orthopedic surgery. Hence, the use of these plants might be beneficial in these patients. Please cite this paper as: Saidi R, Heidari H, Sedehi M, Safdarian B. Evaluating the effect of Matricaria chamomilla and Melissa officinalis on pain intensity and satisfaction with pain management in patients after orthopedic surgery. J Herbmed Pharmacol. 2020;9(4):339-345. doi: 10.34172/jhp.2020.45.

\section{Introduction}

Orthopedic surgeries are among the most painful surgeries. Pain after orthopedic surgeries is mainly due to the nature of the procedure, as major muscular tissues and bones are repaired or reconstructed during these procedures (1). Despite recent advances in post-operative pain management strategies, it is estimated that post- operative pain is not relieved or managed properly in 50\%$75 \%$ of patients (2). Uncontrolled post-operative pain can cause atelectasis, hypoxia and hypercapnia, increased blood pressure and heart rate, myocardial ischemia, dysrhythmia, hyperglycemia, attenuation of immune system, increased platelet adhesion and coagulation, ileus and urinary retention $(3,4)$. Pain can also postpone the

*Corresponding author: Haydeh Heidari,

Email: haydehheidari@gmail.com 
process of bone healing after surgery (5). Post-operative pain is also the most important factor that induces chronic pain after surgeries (6). Furthermore, pain has negative effects on patient satisfaction with quality of cares (7). Therefore, in attempt for improving quality of cares and preventing various adverse effects after orthopedic surgery, proper management of post-operative pain is crucial and should be considered as an important part of post-operative patient cares (8).

The most common strategies for pain management after orthopedic surgeries include the use of oral and intravenous non-steroidal anti-inflammatory drugs (NSAIDs), opioid drugs, and epidural analgesia. Each of these methods have their own specific advantages and disadvantages. The use of opioids is associated with side effects such as nausea, vomiting, constipation, itching, confusion and respiratory depression (9). It is reported that prescription of more opioid drugs is associated with tolerance and which results in more severe pain and less satisfaction with pain management in post-operative patients (10-12). NSAIDs can cause serious side effects in cardiovascular, gastrointestinal and urinary systems $(5,6)$. Epidural analgesia can cause considerable side effects such as epidural hematoma (9). Generally, it can be concluded that, the use of commonly used analgesics at doses needed for complete pain relief, has limited efficacy and considerable side effects (such as respiratory and cardiovascular depression), which can in turn postpone bone healing $(5,13)$. Therefore, it is necessary to search for new methods and strategies that can reduce the dose of analgesic drugs and meanwhile manage patient's pain properly. One of these strategies is the use of complementary and alternative medicine (CAM) (14).

The World Health Organization (WHO) has defined CAM as "diagnostic, therapeutic and preventive measures which are holistic and thus can satisfy unmet needs of patients in the common healthcare systems". CAM is patient-centered and takes all aspects of patient's life into consideration. Nursing is one of the first professions that facilitates the use of CAM, because this profession has a holistic look at patients, and CAM is an appropriate system of care that can fulfil this philosophy. Therefore, CAM is cited as one of the applicable nursing interventions and nurses have the authority to use CAM methods in order to improve various patients' outcomes (15).

One of the main categories of CAM therapies is biological treatment which medicinal herbs are included in this category. Nowadays, various side effects of chemical drugs, low acceptance of chemical drug in population and also limited side effects of medicinal herbs have led to the tendency of general populations, health care professional and researchers to use these herbs more widely. WHO has also emphasized on the use of medicinal herbs (16). In the present study, we used Matricaria chamomilla and Melissa officinalis which are described below:
Matricaria chamomilla L (chamomile) is one of the most commonly used medicinal herbs all over the world (17). There are several therapeutic benefits reported for chamomile, including anti-inflammatory, anti-bacterial, analgesic, accelerating wound healing, sedative, antiallergic and carminative effects (18). These effects are attributed to terpenoids and flavonoids commonly found in chamomile (19). The Food and Drug Administration (FDA) of America has classified chamomile as "generally recognized as safe (GRAS)" (17). Possible interactions of chamomile with medications include its interaction with warfarin (due to the presence of coumarin in chamomile) and cyclosporines (20).

Melissa officinalis is another widely used medicinal herb which has various biological activities. Therapeutic effects of $M$. officinalis include improving mood, reducing anxiety, increasing the level of anti-oxidants in the body, anti-tumor and anti-bacterial activities (21). M. officinalis contains large amounts of Rosmarinic acid, flavonoids and terpenoids, and its analgesic effects are attributed to these substances (22). It is reported that oral prescription of M. officinalis for up to eight weeks is well tolerated (23). There are no reports regarding side effects or toxicity with the use of $M$. officinalis, and this herb is generally considered as a safe medicinal plant (24-26). Posadzki et al in their systematic review reported that $M$. officinalis has pharmaceutical interactions with barbiturates, sedatives and selective serotonin reuptake inhibitors, and should be prescribed with caution in patients receiving these medications (27).

As mentioned above, M. chamomilla and M. officinalis can have suitable analgesic effects. We found no study evaluating the analgesic effects of these medicinal herbs in post-operative orthopedic patients. Thus, this study was conducted with the aim of evaluating the effects of $M$. chamomilla and M. officinalis on pain and satisfaction with pain management in patients after orthopedic surgery.

\section{Materials and Methods}

Study population

This was a randomized controlled trail conducted on 96 patients after lower limb orthopedic surgery in Ayatollah Kashani hospital of Shahrekord, Iran.

Inclusion criteria were patients after lower limb orthopedic surgery, willingness to participate in the study, having normal gag reflex after surgery, age 15 and over, moderate and sever pain intensity (point 4 and above in visual analogue scale; VAS), orientation to time, place and person, not having any underlying disease causing pain, not having the history of sensitivity to $M$. officinalis and $M$. chamomilla, no prescription of warfarin, no prescription of benzodiazepines and sedative drugs, lack of asthma and normal kidney or liver function. Exclusion criteria were unwillingness to continue participation in the study and developing sensitivity to M. officinalis or M. chamomilla. 


\section{Sampling method}

The study sample was calculated 32 patients for each group ( $M$. officinalis, chamomile and control groups) and 96 patients in total). Sampling was conducted with convenience sampling method and patients were randomized to either $M$. officinalis, chamomile, or control group based on the block randomization method. In order to do so, 32 blocks with capacity of 3 patients were made. The number of blocks was determined based on demographic data that could serve as confounding variables, including age group, sex and addiction. There were 4 age groups (15-25, 26-35, 36-45, >45 years), two sexes (male, female) and two modes four addiction (addict, non-addict). Total number of blocks was calculated through the multiplication of the modes of each of these variables: $4 \times 2 \times 2=16$. So, there were 16 blocks with the capacity of 6 patients. Each of these blocks was divided into two blocks with the capacity of 3 patients, so that there were 32 blocks and the capacity of each block was exactly equal to the number of study groups. In order to allocate the participants to the study groups, the first patient entered to each block was allocated to the M. officinalis group, the second patient was allocated to control group and the third patient allocated to chamomile group.

\section{Measurements}

Data were collected using demographic data questionnaire, visual analogue scale for pain and satisfaction with pain management questionnaire. Demographic data questionnaire included questions about age, sex, addiction status, marital status, educational level and medical diagnosis.

VAS is a unidimensional measure of pain which has been widely used in diverse adult populations. This scale is composed of a usually 10 centimeters $(100 \mathrm{~mm})$ horizontal line. Patients can report their pain intensity by a point between the extremes of "no pain at all" and "worst pain imaginable". Simplicity, reliability and validity of this scale have been made it an optimal tool for describing and measuring pain intensity $(28,29)$.

Satisfaction with pain management questionnaire was first developed by Koohestani et al. This questionnaire includes 9 questions with 5-points Likert scale: completely dissatisfied (point=1), dissatisfied (point=2), neither satisfied nor dissatisfied (point=3), satisfied (point=4) and completely satisfied (point $=5$ ). Therefore, the minimum and maximum points acquired from this questionnaire are between 9 and 45 . Point 9 represents completely dissatisfaction with pain management, point 10-18 represents dissatisfaction, points 19-27 represents neither satisfied nor dissatisfied, point 28-36 represents satisfaction with pain management and point 37-45 represents completely satisfaction with pain management. In the study of Koohestni et al, the Cronbach's alpha for this questionnaire evaluated in post-operative patients and was $0.81(30)$.

\section{Interventions}

Melissa officinalis group received $M$. officinalis tea, three times a day during three days. Each time, 1 tea bag containing $1 \mathrm{~g}$ of $M$. officinalis which was produced by Golnoosh Darband Company, put in $150 \mathrm{~mL}$ of boiling water and then consumed by the patient (31).

For chamomile group, 1 tea bag containing $1 \mathrm{~g}$ of $M$. chamomilla put in $150 \mathrm{~mL}$ of boiling water and then prescribed to the patients, 3 times a day during 3 days. These tea bags were produced by Golnoosh Darband Company.

Control group received $1 \mathrm{~g}$ black tea 3 times a day during 3 days. Data were collected before the start of interventions and 30 minutes after prescription of each dose of tea.

Data analysis

After the inclusion of all 96 patients in the study and completion of all interventions, data were analyzed with SPSS software using descriptive (mean and standard deviation) and analytical statistics. For evaluating the efficacy of interventions, the mean score of pain and satisfaction with pain management as well as the mean changes of pain intensity and satisfaction with pain management was compared between study groups using ANOVA and Tukey statistical tests. In all tests the $P$ values $<0.05$ were considered as significant.

\section{Results}

Totally, 96 patients after orthopedic surgery participated in the study. The mean age of participants in the $M$. officinalis group was $36.09 \pm 13.22$, in the chamomile group was $36.28 \pm 12.93$ and in the control group was $36.34 \pm 13.07$ years. Based on age, sex and addiction status, the participants in the three study groups had not significant difference regarding these variables. In each group, 16 patients were addict and 16 patients were nonaddict, and for each age group (15-25, 26-35, 36-45, >45) there were 8 patients in each study group. Demographic data of participants are shown in Table 1.

Before interventions, the mean score of pain intensity was $7.38 \pm 0.18$ in the M. officinalis group, $7.53 \pm 0.19$ in the chamomile group, and $7.31 \pm 0.13$ in the control group. Results of ANOVA test showed no statistically significant different between the three study groups regarding the mean score of pain intensity before interventions $(P=0.659)$.

After 9 steps of intervention, the mean score of pain intensity in the M. officinalis group reduced by $6.0 .3 \pm 0.22$ points, in the chamomile group by $5.93 \pm 0.20$ points and in the control group by $5.68 \pm 0.17$ points. These results show that the mean score of pain intensity reduced in all study groups during the study procedure, but after 
Table 1. Demographic data of participants

\begin{tabular}{|c|c|c|c|c|c|c|c|c|c|c|}
\hline \multirow{2}{*}{ Variable } & & \multicolumn{2}{|c|}{ Melissa officinalis group } & \multicolumn{2}{|c|}{ Chamomile group } & \multicolumn{2}{|c|}{ Control group } & \multicolumn{2}{|c|}{ Total } & \multirow{2}{*}{$\begin{array}{l}\text { P value (between } \\
\text { the } 3 \text { groups) }\end{array}$} \\
\hline & & No. & $\%$ & No. & $\%$ & No. & $\%$ & No. & $\%$ & \\
\hline \multirow{5}{*}{$\begin{array}{l}\text { Location of } \\
\text { surgery }\end{array}$} & Hip & 6 & 18.8 & 3 & 9.4 & 1 & 3.1 & 10 & 10.4 & \multirow{5}{*}{0.242} \\
\hline & Femur & 10 & 31.2 & 13 & 40.6 & 11 & 34.4 & 34 & 35.4 & \\
\hline & Knee & 3 & 9.4 & 5 & 15.6 & 2 & 6.2 & 10 & 10.4 & \\
\hline & Tibia and/or fibula & 8 & 25 & 9 & 28.1 & 16 & 50 & 33 & 34.4 & \\
\hline & Metatarsal bones & 5 & 15.6 & 2 & 6.2 & 2 & 6.2 & 9 & 9.4 & \\
\hline \multirow{2}{*}{$\begin{array}{l}\text { Marital } \\
\text { status }\end{array}$} & Single & 9 & 28.1 & 14 & 43.8 & 15 & 46.9 & 38 & 39.6 & \multirow{2}{*}{0.708} \\
\hline & Married & 23 & 71.9 & 18 & 56.2 & 17 & 53.1 & 58 & 60.4 & \\
\hline \multirow{3}{*}{$\begin{array}{l}\text { Education } \\
\text { level }\end{array}$} & Elementary education & 20 & 62.5 & 15 & 46.9 & 17 & 53.1 & 52 & 54.2 & \multirow{3}{*}{0.816} \\
\hline & High school & 7 & 21.9 & 10 & 31.2 & 9 & 28.1 & 26 & 27.1 & \\
\hline & College education & 5 & 15.6 & 7 & 21.9 & 6 & 18.8 & 18 & 18.8 & \\
\hline
\end{tabular}

steps 1 through 6 of intervention, the mean changes of pain intensity scores in the M. officinalis and chamomile groups were significantly greater than in the control group $(P<0.05)$. The mean changes of pain intensity scores after each step of interventions are compared between groups in Table 2.

Before interventions, the mean score of pain intensity in the $M$. officinalis group was $12.75 \pm 0.42$, in the chamomile group was $12.00 \pm 0.33$, and in the control group was $12.12 \pm 0.33$. Based on the results of ANOVA test, the three study groups had not significant difference regarding the mean score of satisfaction with pain management before interventions $(P=0.37)$.

After 9 steps of intervention, the mean score of satisfaction with pain management in the M. officinalis group increased by $23.96 \pm 0.64$ points, in the chamomile group by $24.46 \pm 0.37$ points and in the control group by $23.81 \pm 0.52$ points. As shown in Table 3, the mean score of satisfaction with pain management was increased during the study interventions, but after steps 1,2 and 3 of interventions, the mean changes of satisfaction with pain management was significantly greater in the M. officinalis and chamomile groups compared with the control group.

Based on the ANOVA and Tukey post hoc tests results, the mean changes of pain intensity and satisfaction with pain management scores were not significantly different between $M$. officinalis and Chamomile groups.

\section{Discussion}

This study was conducted with the aim of evaluating the effects of M. officinalis and M. chamomilla in patients after lower limb orthopedic surgery. The results of the present study showed that, in the first 6 steps of intervention, the mean changes of pain intensity scores in the M. officinalis were significantly greater than those in the control group. As patients were included in the study maximum 24 hours after surgery, it can be concluded that $M$. officinalis was effective in reducing post-operative pain in the first 48-72 hours after surgery. It is important to note that patients were experiencing the most severe pain at this time interval. There are some studies which have evaluated the analgesic effects of M. officinalis in various patients and settings. For example, Mojab et al in 2017 (31) evaluated the effect of $M$. officinalis on the severity of primary dysmenorrhea and reported that the mean changes of pain intensity in the $M$. officinalis group was significantly greater than those in the control group $(P<0.05)$. These results represent the efficacy of $M$. officinalis in reducing pain and are consistent with the results of our study. Zarei et al in their review study reported that, $M$. officinalis have analgesic effect and this effect is mainly due to Rosmarinic acid and flavonoids found in this medical herb (23). Flavonoids regulate the synthesis of prostaglandins and also have effects on opioid receptors in the brain. These actions cause analgesic effects of $M$.

Table 2. Comparing the mean changes of pain intensity scores after each step of interventions between the study groups

\begin{tabular}{|c|c|c|c|c|c|c|c|c|c|}
\hline $\begin{array}{l}\text { Mean changes after each } \\
\text { step of intervention }\end{array}$ & $1^{\text {st }}$ step & $2^{\text {nd }}$ step & $3^{\text {rd }}$ step & $4^{\text {th }}$ step & $5^{\text {th }}$ step & $6^{\text {th }}$ step & $7^{\text {th }}$ step & $8^{\text {th }}$ step & $9^{\text {th }}$ step \\
\hline Melissa & $-0.50 \pm 0.89$ & $-0.93 \pm 0.12$ & $-1.28 \pm 0.1$ & $-2.12 \pm 0.17$ & $-2.31 \pm 0.16$ & $-2.62 \pm 0.18$ & $-5.03 \pm 0.20$ & $-5.53 \pm 0.22$ & $-6.06 \pm 0.22$ \\
\hline Chamomile & $-0.37 \pm 0.08$ & $-0.71 \pm 0.12$ & $-1.06 \pm 0.07$ & $-2.1 \pm 0.13$ & $-2.31 \pm 0.14$ & $-2.65 \pm 0.18$ & $-5.21 \pm 0.19$ & $-5.62 \pm 0.18$ & $-5.93 \pm 0.20$ \\
\hline Control & $-0.03 \pm 0.08$ & $-0.15 \pm 0.07$ & $-0.34 \pm 0.1$ & $-1.4 \pm 17$ & $-1.56 \pm 0.15$ & $-1.93 \pm 0.19$ & $-4.75 \pm 0.16$ & $-5.28 \pm 0.16$ & $-5.68 \pm 0.17$ \\
\hline $\begin{array}{l}P \text { value (Between Melissa } \\
\text { and control groups) }\end{array}$ & $<0.001$ & $<0.001$ & $<0.001$ & 0.007 & 0.003 & 0.02 & 0.55 & 0.63 & 0.44 \\
\hline $\begin{array}{l}P \text { value (between } \\
\text { chamomile and control } \\
\text { groups) }\end{array}$ & 0.004 & 0.002 & $<0.001$ & 0.005 & 0.003 & 0.020 & 0.19 & 0.425 & 0.65 \\
\hline
\end{tabular}


Table 3. Comparing the mean changes of satisfaction with pain management scores after each step of interventions among the study groups

\begin{tabular}{|c|c|c|c|c|c|c|c|c|c|}
\hline $\begin{array}{l}\text { Mean changes } \\
\text { after each step of } \\
\text { intervention }\end{array}$ & $1^{\text {st }}$ step & $2^{\text {nd }}$ step & $3^{\text {rd }}$ step & $4^{\text {th }}$ step & $5^{\text {th }}$ step & $6^{\text {th }}$ step & $7^{\text {th }}$ step & $8^{\text {th }}$ step & $9^{\text {th }}$ step \\
\hline Melissa & $0.9 \pm 0.17$ & $-0.71 \pm 0.12$ & $4.06 \pm 0.34$ & $8.84 \pm 0.5$ & $9.59 \pm 0.48$ & $10.43 \pm 0.53$ & $18.18 \pm 0.51$ & $21.78 \pm 0.64$ & $23.96 \pm 0.64$ \\
\hline Chamomile & $0.93 \pm 0.21$ & $2.31 \pm 0.36$ & $3.93 \pm 0.29$ & $8.87 \pm 0.2$ & $9.75 \pm 0.39$ & $10.65 \pm 0.45$ & $18.68 \pm 0.51$ & $22.5 \pm 0.43$ & $24.46 \pm 0.37$ \\
\hline Control & $-0.21 \pm 0.14$ & $-0.09 \pm 0.1$ & $0.28 \pm 0.21$ & $7.46 \pm 0.3$ & $8.53 \pm 0.39$ & $9.56 \pm 0.44$ & $17.59 \pm 0.57$ & $21.34 \pm 0.52$ & $23.81 \pm 0.52$ \\
\hline $\begin{array}{l}P \text { value (Between } \\
\text { Melissa and control } \\
\text { groups) }\end{array}$ & $<0.001$ & $<0.001$ & $<0.001$ & 0.06 & 0.19 & 0.40 & 0.71 & 0.83 & 0.197 \\
\hline $\begin{array}{l}P \text { value (between } \\
\text { chamomile and } \\
\text { control groups) }\end{array}$ & $<0.001$ & $<0.001$ & $<0.001$ & 0.06 & 0.11 & 0.24 & 0.32 & 0.46 & 0.65 \\
\hline
\end{tabular}

officinalis (26). Guginski et al (32) and Bahmani et al (33) have also reported analgesic the effect for M. officinalis.

In the present study, the mean changes of pain intensity scores in the chamomile group were significantly greater compared with the control group, after the first 6 steps of interventions (after the first 48 hours of intervention). Thus, it can be concluded that $M$. chamomile was effective in reducing post-operative pain in first 2-3 days after surgery, which patients were suffering from most severe pain.

The analgesic effects of $M$. chamomilla have been evaluated in few studies. Modarres et al in 2011 (16) compared the effect of $M$. chamomilla on the severity of primary dysmenorrhea with that of Mefenamic acid and reported that the mean change of pain intensity score in the group receiving $M$. chamomilla was greater than that of the group receiving Mefenamic acid. These results confirm the analgesic effects of $M$. chamomilla and are consistent with the results of our study. Heidaryfard et al in 2015 (34) compared the effect of aromatherapy with M. chamomilla on the severity of labor pain. In this study, aromatherapy with $M$. chamomilla was associated with significant reduction in the severity of labor pain. This study also demonstrates the efficacy of $M$. chamomilla in reducing acute pain. Najafi et al in 2018 (35) evaluated the effect of $M$. chamomilla ointment on the CABG surgery wound pain. They reported that, in days $3^{\text {rd }}, 4^{\text {th }}$, and $7^{\text {th }}$ after surgery, the mean score of pain intensity in the intervention group was significantly lower than that in the control group. These results also represent the analgesic effects of $M$. chamomilla, which is consistent with our results. Shoara et al in 2015 (19) compared the efficacy and safety of topical M. chamomilla oil with diclofenac in patients with knee osteoarthritis. The results of this study showed that there was no significance difference among the group receiving $M$. chamomilla, the group receiving diclofenac and the control group. The results of this study represent that $M$. chamomilla is not efficient in reducing pain, which in inconsistent with the results of our study as well as above mentioned studies.
The results of our study showed no significant difference between $M$. chamomilla and M. officinalis regarding the mean changes of pain intensity scores. Thus, it can be concluded that the efficiency of these two herbs in reducing acute post-operative pain was similar. But, as these two herbs contain different substances which can produce analgesic effects with different mechanisms, it is recommended that more studies be conducted in this regard.

In the present study, the mean changes of satisfaction with pain management score in the $M$. officinalis and chamomile group were significantly greater than those in the control group, only after the first 3 steps of intervention. After that, although the mean score of pain intensity in all group was reduced, but the mean changes of satisfaction with pain management had no significant difference among the three study groups. We found no study evaluating the effect of these medicinal herbs on patient's satisfaction. Since patient's satisfaction is a very important indicator of quality of cares, it is recommended that future studies pay more attention to this important variable.

\section{Conclusion}

This was a randomized controlled trial which evaluated the effect of $M$. officinalis and $M$. chamomilla on pain intensity and satisfaction with pain management in patients after orthopedic surgery. The results of the present study revealed that $M$. officinalis and $M$. chamomilla were effective for reducing pain in the first 48-72 hours after surgery, when patients suffered from most severe pain. These two medicinal herbs, improved satisfaction with pain management only after the first 3 steps of intervention. A review of the literature showed that the number of studies evaluating the analgesic effects of M. officinalis and M. chamomilla were limited and the analgesic effects of these herbs were not approved in all of these studies. So, in order to determine the analgesic effects of M. officinalis and M. chamomilla clearly, more clinical trials are needed. 


\section{Acknowledgements}

This article is a part of a thesis for master degree of medical surgical nursing, which was approved by research deputy of Shahrekord University of Medical Sciences. The researchers are thankful to participants in this study and all who helped us in conducting the study.

\section{Authors' contributions}

All the authors contributed to data collection and preparation of the manuscript. All authors read the final version and confirmed for the publication. SR and $\mathrm{HH}$ did writing proposal, data collection, data analysis and reporting. SM wrote the statistical method, data analysis and reporting, and SB made scientific consultations about patient selection and possible drug interactions with medical herbs used in the study.

\section{Conflict of interests}

The authors report no conflict of interests.

\section{Ethical considerations}

This study was approved by ethics committee of Shahrekord University of Medical Sciences (ethics code: IR.SKUMS.Rec.156) and Iranian Registry of Clinical Trials (identifier: IRCT20170122032101N2). Before any interventions, formal informed consent was obtained from each participant.

\section{Funding/Support}

This study was conducted with financial support of the Research Deputy of Shahrekord University of Medical Sciences (grant No. 2541).

\section{References}

1. Pasero C, McCaffery M. Orthopaedic postoperative pain management. J Perianesth Nurs. 2007;22(3):160-74.

2. Huang N, Cunningham F, Laurito CE, Chen C. Can we do better with postoperative pain management? Am J Surg. 2001;182(5):440-8.

3. Bameshki A, Jahanbakhsh S, Jangjoo A, Zandi H, Fathi M. Evaluation of acute postoperative pain and patient satisfaction in laparotomy, cholecystectomy and herniorrhaphy. Anesthesiol Pain. 2013;3(4):196-201.

4. Han C, Li XD, Jiang HQ, Ma JX, Ma Xl. The use of gabapentin in the management of postoperative pain after total knee arthroplasty: A PRISMA-compliant meta-analysis of randomized controlled trials. Medicine (Baltimore). 2016;95(23):e3883. doi: 10.1097/MD.0000000000003883.

5. Alves CJ, Neto E, Sousa DM, Leitão L, Vasconcelos DM, Ribeiro-Silva $\mathrm{M}$, et al. Fracture pain-traveling unknown pathways. Bone. 2016;85:107-114. doi: 10.1016/j. bone.2016.01.026.

6. Naderi-Nabi B, Haghighi M, Sedighinejad A, Palizkar M, Fathi A, Pourreza M, et al. The effect of premedication with $300 \mathrm{mg}$ Pregabalin on postoperative pain and opioid consumption in patients undergoing elective orthopedic surgery. Anesthesiol Pain. 2014;5(1):29-35.
7. Akyol Ö, Karayurt Ö, Salmond S. Experiences of pain and satisfaction with pain management in patients undergoing total knee replacement. Orthop Nurs. 2009;28(2):79-85.

8. Idvall E, Berg A. Patient assessment of postoperative pain management-orthopaedic patients compared to other surgical patients. Orthop Nurs. 2008;12(1):35-40.

9. Imani F, Rahimzadeh P, Faiz SHR, Sayarifard A, Alebouyeh MR, Entezary SR. Comparison between the effect of 0.2\% and $0.3 \%$ Bupivacaine in fascia iliac block on postoperative pain in patients with femoral or hip fracture. Anesthesiol Pain. 2015;5(4):59-68.

10. Helmerhorst GT, Lindenhovius AL, Vrahas M, Ring D, Kloen P. Satisfaction with pain relief after operative treatment of an ankle fracture. Injury. 2012;43(11):1958-1961. doi:10.1016/j.injury.2012.08.018.

11. Trevino CM, Brasel K. Does opiate use in traumatically injured individuals worsen pain and psychological outcomes? J Pain. 2013;14(4):424-30.

12. Bot AG, Bekkers S, Arnstein PM, Smith RM, Ring D. Opioid use after fracture surgery correlates with pain intensity and satisfaction with pain relief. Clin Orthop Relat Res. 2014;472(8):2542-2549. doi: 10.1007/s11999-014-3660-4.

13. Miller J, Dunion A, Dunn N, Fitzmaurice C, Gamboa M, Myers S, et al. Effect of a brief massage on pain, anxiety, and satisfaction with pain management in postoperative orthopaedic patients. Orthop Nurs. 2015;34(4):227-234. doi:10.1097/NOR.0000000000000163.

14. Soleimani R, Jalali M, Ahmadi R. Attitude of clinical medical students and residents of Guilan University of Medical Sciences about complementary and alternative medicine. Res Med Educ. 2013;5(1):13-21.

15. Zargarzadeh M, Memarian R. Assesing barriers for using of complementary medicine in relieving pain in patients by nurses. Q J Nurs Manag. 2013;1(4):45-53.

16. Modarres, M, Mirmohhamad Ali, M, Oshrieh Z, Mehran A. Comparison of the effect of mefenamic acid and Matricaria camomilla capsules on primary dysmenorrhea. J Babol Univ Med Sci. 2011;13(3):50-8.

17. Srivastava JK, Shankar E, Gupta S. Chamomile: A herbal medicine of the past with bright future. Mol Med Report. 2010;3(6):895-901. doi: 10.3892/mmr.2010.377.

18. Solouki M, Mehdikhani H, Zeinali H, Emamjomeh A. Study of genetic diversity in Chamomile (Matricaria chamomilla) based on morphological traits and molecular markers. Sci Hortic. 2008;117(3):281-7.

19. Pazandeh F, Savadzadeh S, Mojab F, Alavi Majd H. Effects of Chamomile essence on episiotomy healing in primiparous women. J Arak Univ Med Sci. 2008;8(4):364-70.

20. Shoara R, Hashempur MH, Ashraf A, Salehi A, Dehshahri S, Habibagahi Z. Efficacy and safety of topical Matricaria chamomilla L. (chamomile) oil for knee osteoarthritis: a randomized controlled clinical trial. Complement Ther Clin Pract. 2015;21(3):181-7. doi: 10.1016/j.ctcp.2015.06.003.

21. Das M. Chamomile: medicinal, biochemical, and agricultural aspects. CRC Press; 2014. p. 138.

22. Chehroudi S, Fatemi MJ, Saberi M, Salehi SH, Akbari H, Samimi R. Effects of Melissa officinalis L. on reducing stress, alleviating anxiety disorders, depression, and insomnia, and increasing total antioxidants in burn patients. Trauma Mon. 2017;22(4):e33630 
23. Zarei A, Changizi-Ashtiyani S, Taheri S, Hosseini N. A brief overview of the effects of Melissa officinalis L. extract on the function of various body organs. Zanjan J Res Med Sci. 2015;17(7):29-34

24. Akhondzadeh S, Noroozian M, Mohammadi M, Ohadinia S, Jamshidi A, Khani M. Melissa Officinalis extract in the treatment of patients with mild to moderate Alzheimer's disease: a double blind, randomised, placebo controlled trial. J Neurol Neurosurg Psychiatry. 2003;74(7):863-866. doi: $10.1136 /$ jnnp.74.7.863.

25. Alijaniha F, Naseri M, Afsharypuor S, Fallahi F, Noorbala A, Mosaddegh M, et al. Heart palpitation relief with Melissa officinalis leaf extract: double blind, randomized, placebo controlled trial of efficacy and safety. J Ethnopharmacol. 2015;164:378-84.

26. Kennedy D, Scholey AB, Tildesley N, Perry E, Wesnes K. Modulation of mood and cognitive performance following acute administration of Melissa officinalis (lemon balm). Pharmacol Biochem Behav. 2002;72(4):953-64.

27. Posadzki P, Watson L, Ernst E. Herb-drug interactions: an overview of systematic reviews. Br J Clin Pharmacol. 2013;75(3):603-18

28. Bodian CA, Freedman G, Hossain S, Eisenkraft JB, Beilin $Y$. The visual analog scale for pain, clinical significance in postoperative patients. Anesthesiology. 2001;95(6):135661.

29. Hawker GA, Mian S, Kendzerska T, French M. Measures of adult pain: Visual Analog Scale for Pain (VAS Pain), Numeric Rating Scale for Pain (NRS Pain), McGill
Pain Questionnaire (MPQ), Short-Form McGill Pain Questionnaire (SF-MPQ), Chronic Pain Grade Scale (CPGS), Short Form-36 Bodily Pain Scale (SF-36 BPS), and measure of intermittent and Constant Osteoarthritis Pain (ICOAP). Arthrit Care Res. 2011;63(S11):S240-S52.

30. Koohestani H, Baghcheghi N, Rezaei K. Comparison of satisfaction with post-operative pain management and level of functional interferance in addicted and non-addicted patients. J Holist Nurs Midwifery. 2014;24(3):48-55.

31. Mojab F. The effect of Melissa Officinalis extract on the severity of primary dysmenorrhea. Irania J Pharm Res. 2017;16(supl):171-7.

32. Guginski G, Luiz AP, Silva MD, Massaro M, Martins DF, Chaves J, et al. Mechanisms involved in the antinociception caused by ethanolic extract obtained from the leaves of Melissa officinalis (lemon balm) in mice. Pharmacol Biochem Behav. 2009;93(1):10-6.

33. Bahmani M, Shirzad H, Majlesi M, Shahinfard N, RafieianKopaei M. A review study on analgesic applications of Iranian medicinal plants. Asian Pac J Trop Biomed. 2014;7(1):43-53. doi: 10.1016/S1995-7645(14)60202-9.

34. Heidaryfard S, Amir Ali Akbari S, Mojab F, Shakeri N. Effect of Matricaria Camomilla aroma on severity of first stage labor pain. J Clin Nurs Midwifery. 2015;4(3):23-31.

35. Najafi B, Babaei K, Ghesmati F. Studying the Effect of German Chamomile in alleviating CABG surgery wound pain among diabetic patients. J Res Med Dent Sci. 2018;6(1):402-5. 